\title{
Recent Trends in the Characterization of Microbial Exopolysaccharides
}

\author{
K. VENKATA MADHURI ${ }^{1}$ and K. VIDYA PRABHAKAR ${ }^{2 *}$ \\ ${ }^{1}$ Department of Basic Sciences and Humanities, Vignan University, Vadlamudi, Guntur - 522213, India. \\ Department of Biotechnology, Vikrama Simhapuri University, Nellore - 524003, India. \\ ${ }^{*}$ Corresponding author E-mail: kodalividyaprabhakar@gmail.com \\ http://dx.doi.org/10.13005/ojc/300271
}

(Received: April 10, 2014; Accepted: May 20, 2014)

\begin{abstract}
Bacterial polysaccharides that are secreted into the environment are termed as exopolysaccharides (EPSs). Depending on the monosaccharide composition, EPSs can be classified into homo (HoPSs) and heteropolysaccharides (HePSs). HoPSs consist of only one type of monosaccharide, mostly glucose or fructose. In the recent years, EPSs have drawn increasing attention of the researchers worldwide, not only for their thickening, texturizing and viscosifying properties, but also for their health promoting applications. These EPSs constitute an alternative class of biothickeners, which have also been proved to have good emulsifying property, apart from their texture promoting ability in various foods. The biochemical properties of the EPSs depend on the primary structure of the EPSs. Hence it is very important to know the monomeric composition of the EPSs and their gycosidic bonds. HPLC, GC, GC-MS, NMR, Capillary electrophoresis and various bioinformatics tools can be used to determine the chemical characteristics of the EPSs.
\end{abstract}

Key words: Exopolysaccharide; monomeric composition; HPLC; Characterization.

\section{INTRODUCTION}

The polysaccharides are therapeutically potential molecules that have drawn increasing attention of the researchers in last decades due to their constructive physicochemical and biological properties. For instance, starch, pectin guar gum suppress colon cancer incidence to a significant extent (Zhao et al., 2006). Polysaccharides from microbial origin also have been reported to have potential therapeutic applications (Vincent et al.,
2000). Recently, a lot of attention is being paid on the microbial exopolysaccharides (EPSs) due to their health benefits and hence, they have been treated as highly potential molecules (Patricia RuasMadiedo et al, 2002, De Vuyst et al 1999). Depending on the monosaccharide composition, EPSs can be classified into homo (HoPSs) and heteropolysaccharides (HePSs). HoPSs consist of only one type of monosaccharide, mostly glucose or fructose and HePSs are composed of two or more than two types of monosaccharides mostly glucose, 
galactose, mannose and rhamnose. In some EPSs, fucose and hexosamines like glucosamine or galactosamine can also be found in small quantities. The activity of EPS depends on the monosaccharide composition, as the primary structure of proteins (Sutherland, 1994; Cerning, 1995). Most of the researchers concentrate on HePs because of their wide variety of characteristics and biological activities. Biological activities of the EPSs depend on the composition and the monomeric linkages. Hence it is very important to characterize the EPSs chemically and biologically. Various methods that were used in the chemical characterization of the EPSs have been presented in this article.

\section{Methodology of EPS production and characterization \\ Media and cultural conditions}

The amount of EPS production depends on many factors and varies from organism to organism, media composition and cultural conditions. Generally, microorganisms synthesize and secret EPS between their log and stationary phases and varies strain to strain used for EPS production. Lactococcus lactis ssp. Cremoris produces EPS in exponential growth phase and Lactobacillus casei secrets EPS in the early stationary phase. Many food grade bacteria including $L A B$ and propionibacteria release acids lactic acid and propionic acid during their life cycle as a consequence of metabolism in log phase. It can be assumed that these bacteria secrete EPS to survive against acidic conditions during the growth. The probiotic LAB synthesize EPS to endure the gastric acidic conditions and also to adhere to mucosal membrane of the intestine. The production of EPS is also influenced by the oxygen, $\mathrm{pH}$, temperature and media constituents in whom the organism is grown. Petry et al., 2000 observed that the three times increase $\left(110 \mathrm{mg} \mathrm{l}^{-1}\right)$ in EPS production by Lactobacillus delbrueckii subsp. bulgaricus under $\mathrm{pH}$ controlled growth than the amount obtained without $\mathrm{pH}$ control. The amount and mass of EPS is dependent on the type of carbon and nitrogen sources. The Lactobacillus delbrueckii subsp. bulgaricus NCFB 2772 produced less amount and low molecular mass EPS when used fructose in place of glucose as carbon source (Grobben et al. 1997). The carbon/nitrogen ratio plays an important role in the production amount and mass of EPS. The incorporation of complex nitrogen sources like yeast extract, peptone support the bacteria to synthesize EPS in good amounts. But, they may interfere in the quantification and composition analysis of EPS. Where culture medium has increased in complexity, additional purification steps have become necessary to reduce the protein content and other components in the final EPS preparation. The accurate amount and composition analysis can be achieved by using a chemically defined medium supplemented with nutrients like vitamins, nucleic acid bases and amino acids. The viscosity of EPSs is also an important factor because it imparts the viscosity to the nutrient medium used for the growth and production of EPS (Bouzar et al., 1995). So, thorough study of the effect of medium components is required to design large scale production of EPS using fermenter.

\section{Isolation and quantification of EPS}

The most widely used method for crude EPS preparation is precipitation with alcohol (Pham et al., 2000). Two volumes of $10 \%$ trichloroacetic acid (TCA) is added to culture medium to precipitate proteins and kept at room temperature for $1 \mathrm{~h}$. The concentration of TCA and time to keep for protein precipitation are the most important factors that affect the EPS amount. There are the chances to lose EPS If we use more concentration and time for protein precipitation because TCA also precipitates EPS at higher concentrations. The protein contaminants of EPS can be reduced by repeating the TCA a precipitation step twice or thrice. The precipitated proteins are removed by centrifugation; alcohol $(95 \%)$ is added to the supernatant and kept at $4^{\circ} \mathrm{C}$ for overnight. The precipitated EPS is removed by centrifugation and then dialyzed vigorously against water using $12 \mathrm{kDa}$ cut off membrane to remove TCA and small contaminants from EPS fraction. The dialyzed EPS is lyophilized to get dry powder which can be used for further analysis. Acetone has also been used instead of ethanol for precipitation of EPS less frequently.

\section{Quantification}

The EPS can be quantified by turbid metric and colorimetric methods. In turbid metric method, the EPS is expressed as equivalent milligrams of dextran per millimeter. This unit was defined by Gracia-Garibay and Marshal (1991) as the amount 
of polymer that produces same turbidity at $720 \mathrm{~nm}$ as one milligram of dextran (molar mass: $2 \times 10^{3}$ $\mathrm{kDa}$ ) under the same conditions of measurements. But the accuracy this method depends on the degree of the purity of EPS and the contaminants of EPS like proteins or other molecules interfere in the turbidity and gives false results. The colorimetric methods have been widely used for quantification due to their accuracy, sensitivity and simplicity. The anthrone-sulphuric acid method is one of the colorimetric methods used for EPS quantification. Addition of EPS solution to anthrone reagent in 95\% sulphuric gives a characteristic blue color which is a measure for quantitative determination of EPS (Morris, 1948). The anthrone reagent is more expensive and solutions of it in sulphuric acid are not stable and it will not give accurate results for methylated sugars (Dubois et al., 1951). The phenolsulphuric acid method is most widely used for quantification of EPS which is based on orange yellow color produced on addition of phenol and sulphuric acid to the EPS solution (Dubois et al., 1956). However, the phenol-sulphuric acid method measures total carbohydrates present in EPS fraction including low molecular weight carbohydrates that may be present. The other most accurate method for EPS quantification is gel permeation chromatography in a HPLC system, but it is an expensive. In this method, EPS polymers and protein contents are separated based on their size and simultaneously quantified the corresponding elution peaks (Ruas-Madiedo and Gavilan, 2005)

\section{Yield of EPS}

The yield of EPS depends on organism used for production and is also influenced by many environmental factors in which the organism is grown. The reported yields of EPS from some of organisms range from 50 to $350 \mathrm{mg} \mathrm{l}^{-1}$ for Streptococcus thermophilus( Lavender. 2001), 50 to $150 \mathrm{mg} \mathrm{l}^{-1}$ for Lactobacillus delbreuckii ssp. bulgaricus (Bouzar et al., 1995; Petry et al., 2000), 25 to $600 \mathrm{mg} \mathrm{l}^{-1}$ for Lactococcus lactis ssp. cremoris , 50 to $60 \mathrm{mg} \mathrm{l}^{-1}$ for Lactobacillus, 60 to $80 \mathrm{mg} \mathrm{l}^{-1}$ for Lactobacillus paracasei Type $\mathrm{V}$ casei (Cerning, 1995), 280-440 mg l-1 for Lactobacillus rhamnosus, 235 to $320 \mathrm{mg} \mathrm{l}^{-1}$ for Bacillus licheniformis (Larpin et al., 2002) , 110 to $330 \mathrm{mg} \mathrm{l}^{-1}$ for Bacillus coagulans (Kodali \& Sen, 2008), 360 to $400 \mathrm{mg} \mathrm{l}^{-1}$ Bacillus thermoantarciticus (Manca et al., 1996). It was assumed that the EPS production characteristic by thermophilic and mesophilic bacteria is unstable due to the loss of plasmid. But, thermophilic bacteria such as S. thermophilus, Lactobacillus paracase, Lactobacillus delbreuckii do not harbor plasmid and the assumption cannot be applied for these strains and also proved that the EPS synthesis is determined by chromosomal DNA not by plasmid DNA (Germond etal., 2001).

\section{Compositional analysis}

As mentioned above, the HoPSs are composed of only one type of repeating monomer unit and HePSs are composed of two or more different types of monosaccharides. The monosaccharide composition of HePSs is strain specific, and physico-chemical properties and ability to interact with proteins depend on their composition, charge, mass, spatial arrangement, rigidity, linkages between two units and presence of side chains (Cerning. 1995). So the study of structural analysis of polymers is one of the important task before explore into their biological activities. Because of the size, molecular mass and complexity in structure, a combination of several techniques is necessary for complete structural analysis which cannot be performed by a single technique. The compositional analysis of EPSs has been widely performed by high performance liquid chromatography (HPLC) and gas chromatography (GC).

\section{Acid hydrolysis}

The depolymerization of EPSs by acid hydrolysis is the first step in the study of composition and also for further structural determination. Acid hydrolysis is normally done at $100^{\circ} \mathrm{C}$ by using trifluoroacetic acid (TFA), $\mathrm{HCl}$, formic acid or $\mathrm{H}_{2} \mathrm{SO}_{4}$. The concentration of acid, the temperature and time used for acid hydrolysis are important factors and need to be considered carefully. The high concentrations of acid may degrade the released monosaccharides from polymer and low temperatures may cause the incomplete hydrolysis of glycosidic bond between the monomers. Grobben et al. (1997), Ucar et al. 2003 used $1 \mathrm{M} \mathrm{H}_{2} \mathrm{SO}_{4}$ at $100^{\circ} \mathrm{C}$ for $3 \mathrm{~h}$, Levander et al. (2001) used $4 \mathrm{M} \mathrm{HCl}$ at $100^{\circ} \mathrm{C}$ for $45 \mathrm{~min}$, Abdel-Fattah et al. (1974) used $85 \%$ formic acid at $100^{\circ} \mathrm{C}$ for $6 \mathrm{~h}$ and $2 \mathrm{M} \mathrm{TFA}$ at 


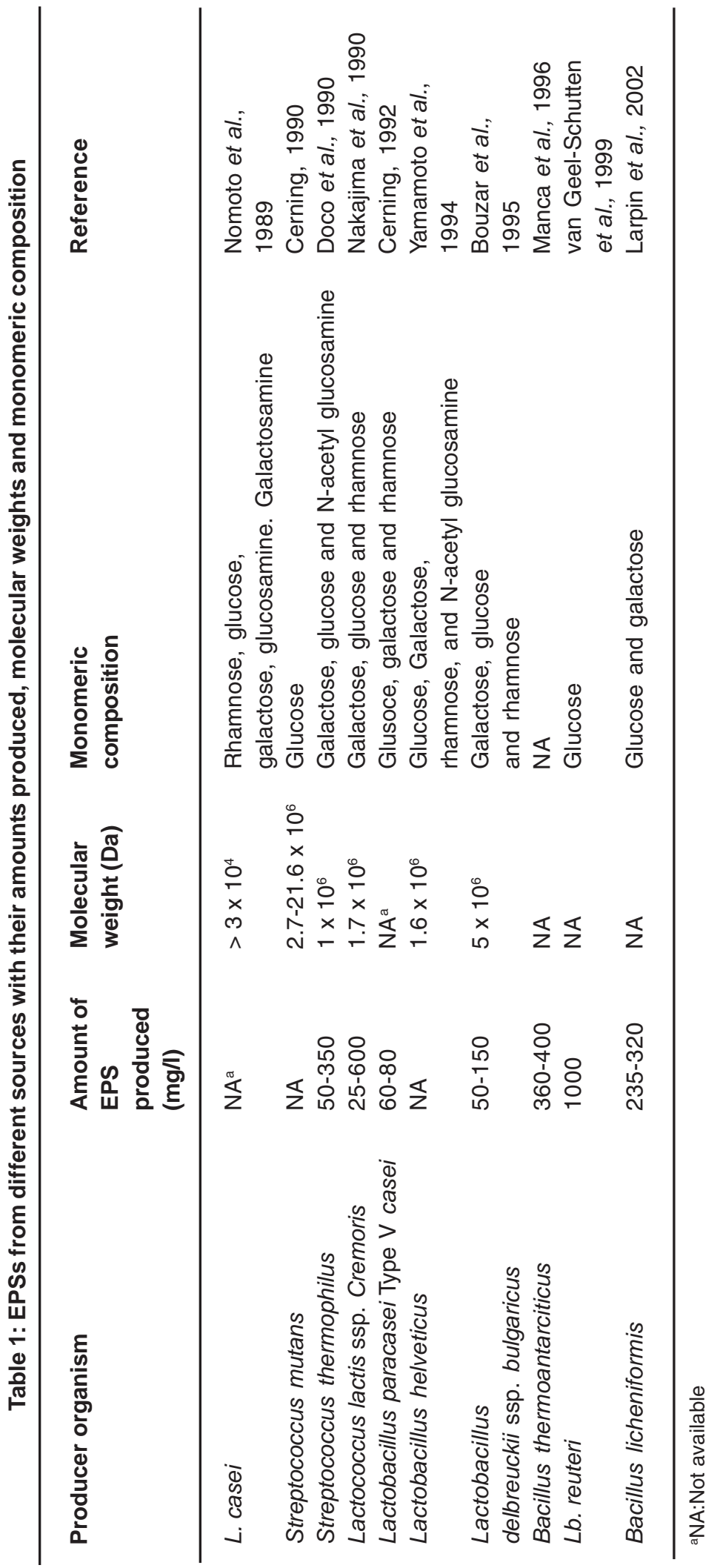


$100^{\circ} \mathrm{C}$ for $6 \mathrm{~h}$ is widely used for acid hydrolysis of EPSs. Levander et al studied the hydrolytic efficiency of $4 \mathrm{M} \mathrm{HCl}$ and $2 \mathrm{MTFA}$ in different intervals and reported that $45 \mathrm{~min}$ in $4 \mathrm{M} \mathrm{HCl}$ gave desirable hydrolysis for the release of aminosugars and more reliable results could be achieved by acid hydrolysis in TFA followed by $\mathrm{HCl}$.

\section{HPLC analysis}

HPLC is can be used for both the qualitative and quantitative analysis of liberated monosaccharides after acid hydrolysis. The high $\mathrm{pH}$ anion exchange (HPAEC) chromatography system with a pulsed amperometric detector (PAD) is widely used for the determination of monomers in foods, beverages, dairy and biotechnological products, vegetable tissues for its simplicity, sensitivity and reliability (Arena et al., 2006). This method can be best used for strongly alkaline solutions and native carbohydrate. This system will not give accurate and reproducible results monomers derived by acid hydrolysis of polysaccharides because acid hydrolysis impart acidic environment to the solution of liberated monomers. HPLC with an ultra violet (UV) detector or refractive index (RI) is an alternative method for quantitative determination of saccharides. Due to low sensitivity and inapplicability to gradient elution, HPLC with RI detector is less commonly used (Arena et al., 2006). The next alternative approach is HPLC with UV detector which is highly sensitive and widely used for analysis and quantification of monomers. Carbohydrates do not absorb UV light and they need pre column derivatization by fluorescent tags to provide UV absorptivity at selected wavelengths. Aromatic amines (Table) have been used as fluorescent tags for derivatizing (labeling) the reducing ends of monosaccharides to enhance the detector sensitivity. Among these, ABEE and AA have been widely reported for derivatization and separated in $\mathrm{C}_{18}$ column under optimized chromatographic conditions (Huang et al., 2000; Tran et al., 2001, Anumula, 2006). The main drawback of the derivatization is inconsistent in yield (\%) and lack of reproducibility. The advantage of the HPLC with derivatization over HPAEC-PAD is sensitivity which is femtomoles for fluorescent tags whereas picomoles for for later one (Cataldi et al., 2000).

\section{GC analysis}

GC is also an important analytical method of analysis of dissociated monosaccharide constituents of polysaccharides and has the advantages of simple instrumentation, high selectivity, quickness and high accuracy. Derivatization of monosaccharides is required due to limited volatility of sugars which renders them into volatilizable and stable derivatives (Lamari et al., 2003). The monosaccharides are either trimethylsilated or converted into alditol acetates in nonaqueous or organic solvents such as pyridine, dimethylsulfoxide (DMSO) for compositional analysis of EPSs. Hexamethyldisilane (HMDS), Trimethylchlorosilane (TMCS), Trimethylsilyl are widely used as trimethylsilating agents that make strongly volatilizable derivatives (Xu et al., 2003;

Table 2 Comparison of biological activity of polysaccharides with their monomeric linkages

\begin{tabular}{|c|c|c|c|}
\hline Polysaccharide source & Glycosidic linkages & Bioactivity & Reference \\
\hline $\begin{array}{l}\text { Phellinus baumii } \\
\text { (Fungas) }\end{array}$ & $(1 \rightarrow 3)$ and $(1 \rightarrow 6)$ & $\begin{array}{l}\text { Antihyperglycemic } \\
\text { activity }\end{array}$ & Hwang et al., 2005 \\
\hline L. sake(Bacterium) & $(1 \rightarrow 6)$ and $(1 \rightarrow 4$ & NR & Robijn et al., 1995. \\
\hline $\begin{array}{l}\text { Arca subcrenata Lischke } \\
\text { (Invertebrate) }\end{array}$ & $(1 \rightarrow 4)$ and $(1 \rightarrow 6)$ & Immuno-stimulatory & He et al., 2007 \\
\hline B. coagulans & $(1 \rightarrow 3),(1 \rightarrow 6)$ and & Antihyperglycemic & Kodali and Sen, Patent \\
\hline $\begin{array}{l}\text { RK-02 (Our bacterial } \\
\text { strain) }\end{array}$ & $(1 \rightarrow 4)$ & $\begin{array}{l}\text { Antioxidant, and } \\
\text { Immunostimulatory } \\
\text { activities }\end{array}$ & $\begin{array}{l}\text { Appl. No. 594/KOL/2009- } \\
\text { of 03/04/2009 }\end{array}$ \\
\hline
\end{tabular}


Wang \& Zhang, 2003). Alditol acetates are prepared by reduction followed by acetylation of monosaccharides. Monosaccharides are reduced with sodium borohydride in anhydrous DMSO and acetylated with 1-Methylimidazole followed by acetic anhydride. Trimethylsilyl derivatives are separated perfectly in GC with moderate polar stationary liquids, and tailing peaks appear if strong polar stationary liquids are used. However, for acetate derivatives, strong polar stationary liquids are the best choice. Both the capillary and packed columns can be used for the separation of derivatized monosaccharides. Capillary columns such as OV-1, OV-225, HP-5, SE-30, Superleco SP 2380, BPB-5 Silar 10C support-coated open tubular (SCOT) glass capillary column widely used and separated using different carriers $\left(\mathrm{He}, \mathrm{N}_{2}, \mathrm{H}_{2}\right)$ and temperature programs (Wang and Fang, 2004). GC attached to mass spectrometer is an efficient and widely applicable method for linkage analysis of methylated polysaccharides. Methylation analysis is an essential step for studying the linkage pattern of sugar residues. Methylation can be performed by either dimsyl sodium/methyl iodide or sodium hydroxide/methyl iodide in which the former methylating agent is widely used (Ciucanu et al., 1984). The methylated EPSs are hydrolyzed partially and then completely. Partial hydrolysis results the cleavage of furanosidic linkages where as the complete hydrolysis results the cleavage of pyranosidic linkages. The pre-methylated EPS is partially hydrolyzed with $90 \%$ formic acid $100^{\circ} \mathrm{C}$ for $6 \mathrm{~h}$ and further hydrolyzed with $2 \mathrm{M} \mathrm{TFA}$ at $100^{\circ} \mathrm{C}$ for $3 \mathrm{~h}$. The resulting products are subjected to reduced and acetylated to get partially methylated alditol acetates (PMAAs) and analyzed by GLCMS and molar ratios are estimated from peak areas and the response factors (Stingele et al., 1999). Nuclear magnetic resonance spectroscopy (NMR) is another powerful technique widely used for establishment the linkage analysis and determination of pyranose/furanose form of monosaccharides (Robijn et al., 1996, Schaffer et al., 2001). Some of the polysaccharides and linkages between their monomer composition have been presented in table 2 .

\section{Capillary zone electrophoresis}

The monosaccharides derivatized with UV absorbing or fluorescent molecules can be efficiently separated by capillary electrophoresis (CE) which is a novel and advantageous technique due to its ease of automation, separation efficiency and it requires small amount of sample. 2-AP, PMP, $A B E E$ have been widely used derivatizing agents for UV detection and 8-Amino-1, 3, 6Naphthalenetrisulfonate (ANTS), 7-Nitro-2, 1, 3Benzoxadiazole-Tagged Methylglycamine (NBD$M G)$ method have been commonly used for fluorescence detection (Honda et al., 2000). For $\mathrm{CE}$, the monosaccharides can be derivatized by either by chemical or enzymatic reactions. Honda et al. derivatized the monosaccharides with 2-AP by reductive amination. Smith and Rassi (1992) analyzed on CE the oligosaccharides derivatized with 2-AP using coated capillary and a buffer of $\mathrm{pH}$ 6. In enzymatic derivatization, two enzymes have been used selectively. One is glucose oxidase which catalyzes the oxidation of glucose, and horse radish peroxidase which catalyzes the oxidation of the non-fluorescent homovanillic acid (HVA) to the fluorescent 2,29-dihydroxy-3,39-dimethoxy biphenyl-5,59-diacetic acid (HVA ) by hydrogen peroxide, produced in the first enzymic reaction. HVA can be monitored by the He/Cd line of LIF detectors. The operating buffer contains both enzymes and HVA, and the sample is introduced by pressure. Chip capillary electrophoresis (CCE) is new micro-total analysis system ( $\mu$-TAS) technique developed rapidly in recent years. In this technique separation channel, reaction container and detector are collected on a chip made by microfabrication technique (Wang and Fang, 2004).

\section{Bioinformatics approaches in the structural analysis of glycans}

Glycomics is a new field of science that deals with the identification and study of all carbohydrate molecules including structural characterization, produced by an organism. The structural characterization refers complete sugar sequence, the monosaccharide stereochemistry, the anomeric configuration and the linkage information. The International Union of Pure and Applied Chemistry (IUPAC) - International Union of Biochemistry and Molecular Biology (IUBMB) "Nomenclature of carbohydrates" specify the three letter code to express the monosaccharide units of glycans. The ring is indicated by an italic $f$ for furanose and $p$ for pyranose form of 
monosaccharides. The interaction between glycans and proteins is crucial to biological function. The bioinformatics approaches provide information of glycan binding proteins (GBPs) so that we can identify and eliminate the disease causing reactions also design the drugs for specific targets. The ancestor of newer oligosaccharide resources is CarbBank, developed in the 1990s, which used a text-oriented IUPAC description of glycans for information retrieval. This was replaced by newer representations, such as LINUCS, used by the GLYCOSCIENCES portal, and GLYDE, an XMLbased description compliant with bioscience standards. A more intuitive approach was taken by the KEGG (Kyoto Encyclopaedia of Genes and Genomes) glycome informatics team in Japan. They developed KegDraw, which produces a graphical description of glycan topology. NMR-derived structural constraints in combination with computational methods are the most frequently used techniques to investigate the dynamic behaviour of the spatial structure of complex carbohydrates. The most often used computational approaches are systematic searches where the relevant torsion angles are systematically changed and the associated energies are calculated, Metropolis Monte Carlo approaches and, increasingly, molecular dynamics simulations. The results are often presented by the 3D structure of the lowest energy conformation or as conformational maps for each glycosidic linkage indicating isoenergetic areas as a function of the torsion angles : and "Several data collections providing energy surfaces are available, the more extensive one, 'GlycoMapsDB'contains about 700 ', " free energy maps obtained from high-temperature simulations of disaccharides. The well-established SWEET-II web interface uses a comprehensive collection of rather crude conformational maps to rapidly generate one realistic glycan conformation from many. The extended alphanumeric nomenclature is required as input. The generated $3 D$ structures are mainly thought to be used as starting points for further refinement using more comprehensive computational techniques. The 'Dynamic Molecules' services the first internet portal which provides an interactive access to set up, perform and analyze molecular dynamic simulations - can take 3D structures created with SWEET-II and provides many features especially devoted to investigating and analyzing the dynamic behavior of complex carbohydrates. The interactive analysis of time dependencies of any interesting degrees of freedom, free energy conformational maps and support for the interpretation of experimental findings mainly derived from NMR spectroscopy are provided by the 'Dynamic Molecules' web interface (Saudhamini et al., .

\section{Molecular mass determination and Purification of EPSs}

The determination of molecular mass of EPS is an important study because the physicochemical and biological properties depend on the molecular mass. The molecular mass of EPSs depends on the strain used for production and monomeric composition of EPSs. Some of the EPS sources and their monomeric composition is represented in the table 1 . It varies from $2.7 \times 10^{6}$ to $2.2 \times 10^{7} \mathrm{Da}$ for strains of Streptococcus mutans, and from $1.5 \times 10^{5}$ to $3.5 \times 10^{6}$ Da for strains of $L b$. reuteri, $4.0 \times 10^{4}$ to $9.0 \times 10^{6} \mathrm{Da}$ for Strep. thermophilus strains (Degeest and De Vust, 1999), $5 \times 10^{5}$ for $L b$ dulbrueckii spp. bulgaricus (Cerning et al., 1986), $1 \times 10^{5}$ to $2 \times 10^{6} \mathrm{Da}$ for Lc. lactis spp. cremoris strains (Nakajima et al., 1990), $2.5 \times 10^{4}$ to $1.4 \times 10^{6}$ Da for $L b$. rhamnosus strains (Pham et al., $2000)$, and $3.5 \times 10^{5} \mathrm{Da}$ for the strain Bifidobacterium longum BB-79 (Roberts et al., 1995) and $1.1 \times 10^{6}$ to $1.2 \times 10^{6} \mathrm{Da}$ for Pseudomonas caryophylli CFR 1705 (Sudhamani et al., 2004), $3 \times$ $10^{5}$ Da for Bacillus thermoantarcticus (Manca et al., 1996), $6 \times 10^{3}$ Da for $P$ acidi propionici (Racine et al., 1991). Various approaches can be used to determine the mass of EPSs. Gel permeation chromatography (GPC) using multiangle laser light scattering (MALLS) detector coupled with RI detector in the HPLC system is widely used method to determine the molecular mass of EPSs (Geresh et al., 2002). Elution of EPS on GPC with a specific column (Sepharose CL 4B or Sephacryl-S 400) which was precalibrated T-series standard dextrans (Sudhamani et al., 2004) or pullulans (Cho et al., 2006) (known molecular weight) is an alternative method of determination of EPS molecular weight .The molecular weight of EPSs can also be determined by using the relative viscosity $\left({ }^{\prime}\right)$ of EPSs by following formula (Brigand \& Denis, 1990).

$$
M=\left({ }_{r}^{1 / 6}-1\right)^{6} / \mathrm{C} \times \mathrm{K}
$$


As mentioned above, the protein contaminants from EPS can be reduced by repeated TCA precipitation and using chemically defined media. EPSs can be further successfully purified by DEAE cellulose chromatography and other columns have also been used for efficient purification. In some reports Sephacryl S-300, S400, S-500, DEAE-Sepharose CL-6B have been used for purification of EPSs (Manca et al., 1996, Sudhamani et al., 2004).

\section{REFERENCES}

1. Zhao, Min Li, Yifan Luo, Weikang Wu. Isolation and structural characterization of an immuno stimulating polysaccharide from fuzi, Aconitum carmichaeli. Carbohydrate Research 2006; 341: 485-491.

2. Vincent ECO, Fang Liu. Immunomodulation and Anti-Cancer Activity of PolysaccharideProtein Complexes. Current Medicinal Chemistry 2000; 7: 715-729.

3. Ruas-Madiedo P, Hugenholtz J, Zoon P. An overview of the functionality of exopolysaccharides produced by lactic acid bacteria. International Dairy Journal 2002; 12: 163-171.

4. De Vuyst L, Degeest B. Hetero polysaccharides from lactic acid bacteria. FEMS Microbiology Reviews 1999; 23: 153177.

5. Sutherland IW. Structure- Function relationships in microbial exopolysaccharides. Biotechnology Advances 1994; 12: 393-448.

6. Cerning J. Production of exopolysaccharides by lactic acid bacteria and dairy propionibacteria. Le Lait Diary Science and Technology 1995; 75: 463-472.

7. Petry S, Furlan S, Crepeau MJ, Cerning J, Desmazeaud M. Factors Affecting Exocellular Polysaccharide Production by Lactobacillus delbrueckii subsp. bulgaricus Grown in a Chemically Defined Medium. Applied and Environmental Microbiology 2000; 66: 34273431.

8. Grobben GJ, van Casteren WHM, Schols HA, Oosterveld A, Sala G, Smith MR, Sikkema J, de Bont JAM.Analysis of the exopolysaccharides produced by Lactobacillus delbrueckii subsp. bulgaricus NCFB 2772 grown in continuous culture on glucose and fructose. Applied Microbiology and Biotechnology 1997; 48: 516-521.
9. Bouzar F, Cerning J, Desmazeaud M. Exopolysaccharide production in milk by Lactobacillus delbrueckii subsp. bulgaricus CNRZ 1187 and by two colonial variants. Journal of Dairy Science 1996; 79: 205-211.

10. Pham PL, Dupont I, Roy D, Lapointe G, Cerning J. Production of Exopolysaccharide by Lactobacillus rhamnosus $\mathrm{R}$ and Analysis of Its Enzymatic Degradation during Prolonged Fermentation. Applied and Environmental Microbiology 2000; 66: 23022310.

11. Morris D L. Quantitative determination of carbohydrates with Dreywood's anthrone reagent. Science 1948; 107:254-5.

12. Dubois M, Gilles K, Hamilton JK, Rebers PA, Smith F. A Colorimetric Method for the Determination of Sugars Nature 1951; 168 : 167.

13. Dubois M, Gilles KA, Hamilton JK, Pebers PA, Smith F. Colorimetric method for determination of sugar and related substances. Analytical Chemistry 1956; 28 : 350-356.

14. Ruas-Madiedo P, Reyes-Gavilan CG. Invited Review: Methods for the Screening, Isolation, and Characterization of Exopolysaccharides Produced by Lactic Acid Bacteria. Journal of Dairy Science 2005; 88:843-856.

15. Levander FMS, Peter Radstrom. Small-scale analysis of exopolysaccharides from Streptococcus thermophilus grown in a semidefined medium. BMC Microbiology 2001; 1 : 23

16. Larpin S, Sauvageot N, Pichereau V, Laplace JM, Auffray Y. Biosynthesis of exopolysaccharide by a Bacillus licheniformis strain isolated from ropy cider. International Journal of Food Microbiology 2002; 77(1-2):1-9.

17. Manca MC, Lama L, Improta R, Esposito E, 
Gambacorta A, Nicolaus B. Chemical Composition of Two Exopolysaccharides from Bacillus thermoantarcticus. Applied and Environmental Microbiology 1996; 62: 32653269.

18. Germond JE, Delley M, D Amico N, Vincent SJ. Heterologous expression and characterization of the exopolysaccharide from Streptococcus thermophilus Sfi39. European Journal of Biochemistry 2001; 268 : 5149-5156.

19. Huang Z, Prickett T, Potts M, Helm R. The use of the 2-aminobenzoic acid tag for oligosaccharide gel electrophoresis. Carbohydrate Research 2000; 328: 77-83.

20. Tran N, Daali Y, Cherkaoui S, M. Taverna M, Neeser J, Veuthey J. J. Chromatogr. A 929 (2001) 151. (Tran NT, Daali Y, Cherkaoui S, Taverna M, Neeser JR, Veuthey JL. Routine o-glycan characterization in nutritional supplements - a comparison of analytical methods for the monitoring of the bovine kappa-casein macropeptide glycosylation. Journal of Chromatography A 2001; 929: 151163.

21. Kalyan R, Anumula. Advances in fluorescence derivatization methods for highperformance liquid chromatographic analysis of glycoprotein carbohydrates. Analytical Biochemistry 2006; 350: 1-23.

22. Cataldi TR, Margiotta G, lasi L, Di Chio B, Xiloyannis C, Bufo SA. Determination of sugar compounds in olive plant extracts by anion-exchange chromatography with pulsed amperometric detection. Analytical Chemistry 2000; 72: 3902-3907.

23. Xu, B., Xiao, G., Ding, X., Chin. J. Chromatogr. 2003; 21: 410

24. Wang, L., Zhang, Z. J. Dalian Nat. Univ. 2003; $5,54$.

25. Ciucanu I, Kerek F. A Simple and rapid method for the permethylation of Carbohydrates. Carbohydrate Research 1984; 131: 209-217.

26. Stingele F, Vincent SJF, Faber EJ, Newel JW, Kamerling JP, Neeser JR. Introduction of the exopolysaccharide gene cluster from Streptococcus thermophilus Sfi6 into Lactococcus $\beta$ MG1363: production and characterization of an altered polysaccharide. Molecular Microbiology 1999; 32: 1287-1295.

27. Robijn WG, Hans LJ, Dick JC, Haas H, Johannis PK, Johannes FGV. Structural studies of the exopolysaccharide produced by Lactobacillus paracasei 34- 1. Carbohydrate Research 1996; 285: I29- 139.

28. Schaffer C, Tali S, Christian R, Paul K, Sonja Z, Paul M, Sharon N. Purification and structure elucidation of the $\mathrm{N}$-acetylbacillosaminecontaining polysaccharide from Bacillus licheniformis ATCC 9945. European Journal of Biochemistry 2001; 268: 857-864.

29. Honda S, Okeda J, Iwanaga H, Kawakami S, Taga A, Suzuki S, Imai K. Ultramicroanalysis of reducing carbohydrates by capillary electrophoresis with laser-induced fluorescence detection as 7-nitro-2,1,3benzoxadiazole-tagged $\mathrm{N}$-methylglycamine derivatives. Analytical Biochemistry 2000; 286: 99-111.

30. Smith, J.T., Rassi E.I., J. High Resolut. Chromatogr. 1992; 15, 573

31. Sudhamani SR, Tharanathan RN, Prasad MS. Isolation and characterization of an extracellular polysaccharide from Pseudomonas caryophylli CFR 1705. Carbohydrate Polymers 2004; 56: 423-427.

32. Cerning J, Bouillanne C, Desmazeaud MJ, Landon M. Isolation and characterization of exocellular polysaccharide produced by Lactobacillus bulgaricus. Biotechnology Letters 1986; 8: 625-628.

33. Nakajima H, Toyoda S, Toba T, Itoh T, Mukai $\mathrm{T}$, Kitazawa $\mathrm{H}$, Adachi S. A novel phosphopolysaccharide from slime forming Lactococcus lactis subspecies cremoris SBT 0495. Journal of Dairy Science 1990; 73: 1472-1477.

34. Degeest B, De Vuyst L. Indication that the Nitrogen Source Influences Both Amount and Size of Exopolysaccharides Produced by Streptococcus thermophilus LY03 and Modelling of the Bacterial Growth and Exopolysaccharide Production in a Complex Medium. Applied and Environmental Microbiology 1999; 65: 2863-2870.

35. Sudhamani SR, Tharanathan RN, Prasad MS. Isolation and characterization of an extracellular polysaccharide from 
Pseudomonas caryophylli CFR 1705. Carbohydrate Polymers 2004; 56: 423-427.

36. Roberts CM, Fett WF, Osman SF, Wijey C, OOonnor JV, Hoover DG. Exopolysaccharide production by Bifidobacterium longum BB79. Journal of Applied Bacteriology 1995; 78: 463-468.

37. Geresh S, Adin I, Yarmolinsky E, Karpasas M. Characterization of extra cellular polysaccharide of Porphyridium sp.: molecular weight determination and rheological properties. Carbohydrate polymers 2002; 50: 183-189.

38. Cho EJ, Oh JY, Chang HY, Yun JW. Production of exopolysaccharides by submerged mycelial culture of a mushroom Tremella fuciformis. Journal of Biotechnology 2006; 127: 129-140.
39. Brigand G, Denis A, Grall M, Lecacheux D. Insight into the Structure of Pectin by High Performance Chromatographic Methods. Carbohydrate Polymers 1990; 12: 61-77

40. Nakajima H, Toyoda S, Toba T, Itoh T, Mukai $\mathrm{T}$, Kitazawa $\mathrm{H}$, Adachi $\mathrm{S}$. A novel phosphopolysaccharide from slime forming Lactococcus lactis subspecies cremoris SBT 0495. Journal of Dairy Science 1990; 73: 1472-1477.

41. Kodali and Sen, Patent Appl. No. 594/KOL/ 2009-of 03/04/2009

42. Kodali VP and Ramkrishna Sen, Antioxidant and free radical scavenging activities of an exopolysaccharide from a probiotic bacterium, Biotechnology Journal, 2008; 2: 245-251. 